\title{
Social correlates of malnutrition risk in naturally occurring retirement communities in Ontario, Canada
}

Older adults are the fastest-growing demographic group in Canada ${ }^{(1)}$. Most older adults want to age-in-place within their communities $^{(2)}$. One-third of these community-dwelling older adults in Canada are at malnutrition risk, the risk of poor dietary intake and nutritional status, with consequences including increased frailty, decreased quality of life, increased hospitalization, and higher mortality rates ${ }^{(3)}$. It is therefore important to identify correlates of malnutrition risk, especially as they may be root causes of poor food intake and are amenable to intervention.

Participants were recruited from seven naturally occurring retirement communities in the province of Ontario, Canada. Demographic data, social measures, and malnutrition risk scores were collected. Individuals were screened for malnutrition risk using Seniors in the Community: Risk Evaluation for Eating \& Nutrition (SCREEN)-14, a valid and reliable tool for screening for malnutrition risk in community-dwelling older adults. Scores range from 0-64, with scores less than 54 indicating malnutrition risk. Social networks were measured using the Lubben Social Network Scale (LSNS) a valid and reliable tool for measuring social networks and engagement; scores range from 0-30, with higher scores indicating greater social engagement. Social Support was measured using the Positive Social Interaction subscale of the MOS Social Support Survey (MOS); scores range from 0-25, with higher numbers indicating greater social support. The MOS has demonstrated validity and reliability. Social participation was measured by asking individuals how frequently they were involved in community activities. Descriptive statistics were calculated for all variables. Spearman's rho and Pearson's correlation coefficient were calculated where appropriate.

In total, 128 individuals were screened. SCREEN-14 scores ranged from 19 to 62 , with a mean score of 45 (SD $=7.9)$. LSNS ranged from 0 to 30, with a mean score of $15(\mathrm{SD}=6.4)$. MOS scores ranged from 3 to 20, with a mean score of $12(\mathrm{SD}=4.4) .44 .4 \%$ of respondents never participated in community activities, $12.6 \%$ participated less than once a month, 7.4\% twice a month, 16.3\% once a week, and $19.6 \%$ two to three times a week. Age $(r=.302, p=.001)$, household income $(\rho=.255, p=.009)$, LSNS $(\rho=.280$, $p=.001)$ and $\operatorname{MOS}(\rho=.294, p=.001)$ were all significantly associated with SCREEN-14 score. Frequency of participation in community activities was not associated with SCREEN-14 score.

Several social factors are associated with malnutrition risk scores among residents in retirement communities in Ontario, Canada. Participants who were younger, who had lower incomes, lower social engagement and less social support had lower SCREEN-14 scores, indicating that these individuals were at increased malnutrition risk. Addressing social factors may therefore help to improve malnutrition risk scores in members of this population.

\section{References}

1. Statistics Canada (2018) Population Projections for Canada (2018 to 2068) [Available at: https://www150.statcan.gc.ca/n1/pub/91-520-x/ 91-520-x2019001-eng.htm

2. National Institute on Ageing (2019) Enabling the Future Provision of Long-Term Care in Canada

3. Ramage-Morin PL, Gilmour H, Rotermann M (2017) Health Reports 28(9), 17-27 